Bestandsaufnahme der Massnahmen gegen weibliche Genitalverstümmelung in der Schweiz in den Bereichen Prävention, Unterstützung und Schutz

\title{
Trotz guter Beispiele im Gesundheitsbereich - Handlungsbedarf besteht weiterhin
}

In der Schweiz sind schätzungsweise rund 13000 Frauen und Mädchen von Genitalverstümmelung (FGM) betroffen oder bedroht [1]. In den letzten Jahren wurden daher Anstrengungen unternommen, um Betroffene zu beraten und zu unterstützen sowie (potentiell) Gefährdete zu schützen. In einer Bestandsaufnahme [2] hat Terre des Femmes Schweiz diese diversen Aktivitäten zusammengetragen. Im Gesundheitsbereich gibt es modellhafte Beispiele, doch gilt es auch noch immer, Lücken zu schliessen [3].

Marisa Birri

Korrespondenz:

Marisa Birri

Terre des Femmes Schweiz

Standstrasse 32

CH-3014 Bern

m_birri[at]terre-des-femmes.ch

www.terre-des-femmes.ch
«Practices with potential» im

\section{Gesundheitsbereich}

In einigen grösseren Frauenkliniken der Schweiz werden seit rund zehn Jahren regelmässig Erfahrungen mit beschnittenen Patientinnen gemacht, dies mehrheitlich im Zusammenhang mit Schwangerschaft und Geburt. In diesen Kliniken arbeiten vereinzelt Fachpersonen, die sich auf FGM spezialisiert haben und den Patientinnen adäquate Behandlung und Unterstützung bieten können. Dabei arbeiten diese eng mit Dolmetscherinnen und interkulturellen Vermittlerinnen zusammen.

In einigen wenigen Spitälern wurde das Thema sogar institutionell verankert. Beispielsweise gibt es im Frauenspital des Inselspitals Bern Gynäkolo-

\section{In einigen wenigen Spitälern} wurde das Thema sogar institutionell verankert.

ginnen, die auf die Bedürfnisse von beschnittenen Frauen spezialisiert sind. Zudem führen Hebammen gemeinsam mit interkulturellen Vermittlerinnen mit allen beschnittenen Frauen im Rahmen der regulären Nachkontrolle nach der Geburt ein «Präventionsgespräch», das auf einem speziellen Fragenkata$\log$ basiert. Auch die Abteilung Gynäkologie und Geburtshilfe des Spitals Neuenburg fällt positiv auf. Sie hat im Rahmen der kantonalen Strategie zur Überwindung von FGM zusammen mit der Familienplanungsstelle einen Handlungsleitfaden erarbeitet. Dieser definiert die Rolle jeder involvierten Person sowohl innerhalb als auch ausserhalb des Spitalsettings und legt den Ablauf und die Zusammenarbeit im Zusammenhang mit einer beschnittenen Patientin fest. Schliesslich kann auch die multidisziplinäre

\section{MGF en Suisse - un état des lieux}

Malgré de bons exemples dans le domaine de la santé il y a toujours un besoin d'action.

De nombreux efforts ont été entrepris au cours des dernières années pour conseiller, soutenir et protéger les filles et femmes concernées par les mutilations génitales féminines (MGF) en Suisse. Dans le cadre d'un état des lieux, Terre des Femmes Suisse a recueilli ces différentes activités et montré des exemples modèles ainsi que les lacunes.* Alors que ce sont notamment la gynécologie et l'obstétrique qui font l'expérience depuis longtemps avec des femmes excisées, le sujet n'est institutionnalisé que dans quelques hôpitaux, que ce soit par l'application d'un protocole de prise en charge ou la mise en place d'un groupe de travail interdisciplinaire au sein de I'hôpital. De même, les MGF devraient aussi être abordées de manière exhaustive et d'un point de vue pratique au cours de la formation des médecins. Par ailleurs, les offres spécifiques aux MGF ne sont pas disponibles dans tout le pays. Beaucoup dépend de l'engagement individuel et une intégration dans les processus de travail ne se fait pas systématiquement. II y a également un potentiel important dans la prévention primaire et secondaire, qui se retrouve plutôt au second plan dans le domaine de la santé. Les MGF devraient alors être reconnues davantage comme une forme de maltraitance des enfants et une violence domestique et il faudrait réagir de manière similaire en conséquence. II y a encore un besoin de discussion par rapport à la mise en pratique ainsi qu'au rôle qui revient notamment aux pédiatres.

\footnotetext{
* Pour les résultats détaillés, voir MGF en Suisse. Etat des lieux des mesures de prévention, de soin et de protection en matière de mutilations génitales féminines en Suisse, Résumé, Berne 2014, www.terre-des-femmes.ch/fgm/unser-engage ment-gegen-fgm/bestandesaufnahme
} 


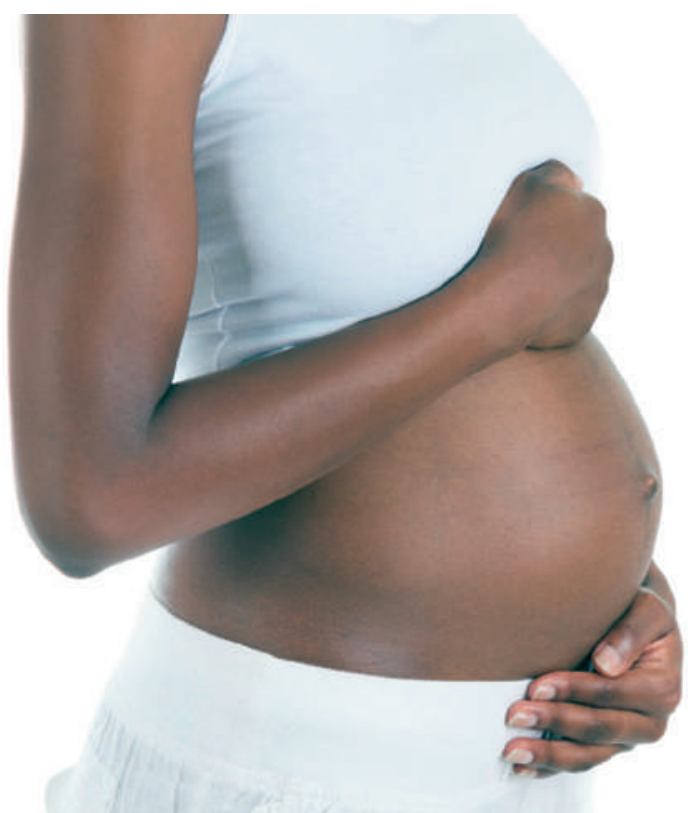

In der Schweiz werden Gesundheitsfachpersonen oder Ärztinnen und Ärzte meist im Rahmen von Schwangerschaft und Geburt mit Genitalverstümmelung konfrontiert.

Zusammenarbeit innerhalb des Universitätsspitals Genf angeführt werden: Die spezialisierte Sprechstunde der Abteilung Gynäkologie/Geburtshilfe bietet eine medizinische, psychophysische und chirurgische Versorgung für beschnittene Frauen. Die Sprechstunde arbeitet eng mit einer multidisziplinären Gruppe «FGM» (Ethnopsychiatrie, Sexologie, Pädiatrie, Rechtsmedizin, Medizinethnologie, Gewaltprävention etc.), mit der Abteilung sexuelle Ge- kommt massgebende Bedeutung zu. Denn die Tochter einer beschnittenen Mutter ist potentiell gefährdet, zum Schutz des Mädchens muss unbedingt entsprechend gehandelt werden.

Der Gesundheitsbereich sieht seine primäre Rolle in der Unterstützung und Versorgung von bereits beschnittenen Frauen und Mädchen. Die primäre und sekundäre Prävention scheint eher zweitrangig, obwohl gerade der Gesundheitsbereich entscheidende Anknüpfungspunkte bietet, um auch Präventionsgespräche zu führen. Auch können Gesundheitsfachpersonen mit Verdachtsfällen konfrontiert sein, wobei es gilt, FGM vermehrt als eine Form von Kindesmissbrauch und häuslicher Gewalt wahrzunehmen und analog vorzugehen. In Bezug darauf, wie dies in der Praxis umgesetzt werden könnte und welche Rolle dabei insbesondere den Pädiaterinnen und Pädiatern zukommt, besteht noch Diskussionsbedarf.

Informationen darüber, ob und wie FGM in der Schweiz auch in der Ausbildung von Ärztinnen und Ärzten Thema ist, konnten bei den medizinischen Fakultäten leider nicht eruiert werden. Hingegen ist FGM in einigen Hebammen- und Pflegeausbildungen sowie Ausbildungen zum/zur Berater/in der sexuellen und reproduktiven Gesundheit bereits seit längerem Teil der obligatorischen Grundausbildung. Es müssen nicht alle Gesundheitsfachpersonen zu FGMExperten werden. Eine minimale Sensibilisierung im Rahmen der Grundausbildung würde jedoch bedeuten, dass alle über Handlungsoptionen bei Komplikationen im Zusammenhang mit FGM oder einer drohenden Beschneidung informiert sind.

\section{Der Gesundheitsbereich bietet auch Anknüpfungspunkte für die primäre und sekundäre FGM-Prävention.}

sundheit und Familienplanung sowie mit der Sprechstunde gynäkologische Psychosomatik und Sexualmedizin zusammen.

\section{Handlungsbedarf}

Die Bestandsaufnahme zeigt auf, dass zwar FGM-spezifische Angebote im Gesundheitsbereich existieren, dass die qualitativen Unterschiede dabei jedoch enorm sind und dass von einer Flächendeckung nicht die Rede sein kann. Sehr oft gehen die Bemühungen auf einzelne engagierte Personen zurück, zu selten erfolgt eine systematische Einbettung in Arbeitsabläufe. Auch die Vernetzung und Zusammenarbeit mit medizinischen Fachpersonen jenseits der Gynäkologie und Geburtshilfe gestaltet sich schwierig. Doch gerade dem Follow-up nach einer Geburt und dem Informationsfluss an die Pädiatrie, die Mütterberatungs- und Familienplanungsstellen

\section{Referenzen}

1 Laut Schätzungen des Bundesamtes für Gesundheit 2013.

2 Diese Bestandsaufnahme ist Teil der Grundlagenarbeit der Nationalen Arbeitsgruppe gegen Mädchenbeschneidung (AG FGM). Vgl. hierzu www.bag.admin. ch/themen/gesundheitspolitik/07685/12512/14074/ index.html?lang=deAG. Auf der Webseite sind auch die Umfrage bei Fachpersonen von Unicef Schweiz von 2013 sowie die Handlungsempfehlungen vom Schweizerischen Kompetenzzentrum für Menschenrechte von 2014 erschienen.

3 Für die ausführlichen Ergebnisse siehe Terre des Femmes Schweiz, FGM in der Schweiz. Bestandesaufnahme über die Massnahmen gegen weibliche Genitalverstümmelung in der Schweiz in den Bereichen Prävention, Unterstützung und Schutz, Kurzfassung, Bern 2014. www.terre-des-femmes.ch/fgm/ unser-engagement-gegen-fgm/bestandesaufnahme 